\title{
MEASURING LOGARITHMIC SIGNAL CONVERTER FOR MAGNETIC TRACKING SYSTEMS
}

\author{
Roman Holyaka, Dr. Sc., Prof., Tetyana Marusenkova, Ph.D., Ass. Prof., \\ Dmytro Fedasyuk, Dr. Sc., Prof. \\ Lviv Polytechnic National University, Ukraine, e-mail: holyaka@yahoo.com;
}

\begin{abstract}
The work deals with the problem of signal conversion in magnetic tracking systems. Magnetic tracking systems are a novel development trend of navigation sensors within the concepts of the Internet of Things and virtual and augmented reality. In contrast to optical tracking systems, magnetic ones do not suffer from occlusions. In comparison with tracking systems built upon inertial sensors, they are not susceptible to bias drift and provide better accuracy. Magnetic tracking technology is based on calculating the position of objects upon the dynamic measurement of the reference magnetic field vectors. The reference magnetic fields are formed by arrays of actuator coils in the low-frequency electromagnetic radiation spectrum. Those who implement a magnetic tracking system have to ensure noise-immune measurements of signals coming from sensor coils in a wide dynamic measurement range. The range changes from microvolts for distances of several meters in couples "actuator-sensor" to hundreds of millivolts in the case if the distances in "actuator-sensor" couples reduce to several centimeters. Thus, one requires signal converters able to provide highly noise-immune measurements in a dynamic measurement range covering six orders of magnitude. The work presents the results of development, simulation, and investigation into a signal converter for magnetic tracking systems, whose novelty consists in combining the methods of logarithmic amplification and synchronous demodulation of the output signals of the sensor coils. The main nodes of the developed signal converter are a control unit, a logarithmic amplifier, a synchronous demodulator, a low-pass filter, an actuator driver and an analog-to-digital converter. Voltage logarithm compression has been performed upon volt-ampere characteristics of semiconductor $p-n$ junctions. The synchronous demodulator provides a high level of selection of the useful signal out of electromagnetic noise. The results presented in this paper are part of our complex research work related to the development of the Magnetic Tracking System Integrated Development Environment (MTS-IDE). The latter is being developed by a team of scholars within different projects and is aimed at enhancing the efficacy of parametric optimization and synthesizing firmware of embedded systems implementing integrated magnetic tracking sensors. Simulation and experiments have shown that the dynamic range of noise-immune signal measurement using the developed converter covers six orders of magnitude, from $1 \mathrm{E}-6 \mathrm{~V}$ to $1 \mathrm{~V}$. Investigation into functionality were conducted by oscillograph methods. The characteristics of the proposed solution were measured by the above-mentioned MTS-IDE. The obtained results are of key importance for further improvement of magnetic tracking systems, particularly, for their noise-immune measurement volume expansion.
\end{abstract}

\section{Keywords}

Spatial Navigation, Magnetic Tracking, Virtual Reality Devices, Analog Front End, Signal Compression, Logarithmic Converter

\section{Introduction}

Magnetic tracking systems are a novel development trend of sensors for object navigation that conforms with Virtual Reality (VR) and Augmented Reality (AR) concepts [1]. Magnetic tracking technology relies on calculating the spatial position of an object being tracked based on the measurement of the reference magnetic field vectors in the lowfrequency electromagnetic radiation spectrum. Hence, the technology is also referred to as Electromagnetic Tracking (EMT) [2].

Measurements are carried out using coils that form a system of coupled actuators and sensors. Actuator coils induce AC (Alternative Current) magnetic fields whereas sensor coils generate an electromotive force (AC voltage) caused by the fields. The informative signals that characterize the mutual location in couples "actuator - sensor" are described by functional dependencies whose main arguments are the magnetic coupling coefficient in the couple and the quantities to be measured. The latter is the distance between the coils and the angle between them. The spatial position is calculated upon mathematical models of the distribution of the formed magnetic fields. The models are synthesized upon theoretical assumptions and experimental results.

A substantial advantage of magnetic tracking systems over optical ones is the fact that they do not require a direct line of sight. In contrast to inertial tracking systems, which utilize inertial measurement units, magnetic tracking systems do not suffer from bias drift intrinsic for accelerometers [3] and gyroscopes [4] and are able to provide high-precision measurements of the coordinates of the sensors in a coordinate frame formed by the actuator array. 
Modern solutions for magnetic tracking include AR IoT, a universal framework that conforms with the AR and Internet of Things (IoT) concepts [5]; data fusion in medical imaging devices [6]; surgical instruments navigation in medical equipment [7]; biomedical engineering technologies including those for monitoring orofacial kinematics [8]; flexible robot mechatronics tools [9], etc. Magnetic tracking systems can be implemented upon specialized or unified coils [10]. Mainly universality of magnetic tracking systems is provided by 3D (3-Dimension) small-size coils [11]. A combination of magnetic and inertial tracking is presented in $[12,13]$.

\section{Shortcomings}

When designing a magnetic tracking system one has to ensure noise-immune measurement of signals coming from sensor coils in a wide dynamic measurement range (typically, voltages are measured). The range changes from microvolts for distances of several meters in couples "actuator-sensor" to hundreds of millivolts in the case if the distances in "actuator-sensor" couples reduce to several centimeters. Therefore, one requires signal converters able to provide highly noise-immune measurements in a dynamic measurement range covering six orders of magnitude. Thus, the solution to the above-mentioned important problem of highly-noise immune measurements in the stated range would give rise to enhancement of operating characteristics of magnetic tracking systems.

\section{Goal of the Study}

The work is aimed at development, simulation and experimental study of a signal converter for magnetic tracking systems, which would provide highly noise-immune signal measurements in a measurement range covering six orders of magnitude and thus allow expansion of measurement volumes without loss of accuracy in measuring coordinates.

\section{Measuring Logarithmic Signal Converter}

Research results in the field of the magnetic tracking are related to position and orientation estimates in magnetic tracking systems [14], development of magnetic tracking systems for near-field scanning [15], calibration of tracking systems for dynamic field distortion compensation [16], positional accuracy assessment and error modeling for magnetic tracking systems [17], etc.

The measurement principle in magnetic tracking systems consists in calculation of the object spatial position. Precisely, arrays of coordinates and angles of sensors in some given coordinate system are to be found. In the origin of this coordinate frame, the actuator forms a low-frequency magnetic field. Usually one-, two- or three-dimensional (3D) small-size coils serve as sensors and actuators. Approximately, the maximum value of the output voltage $\mathrm{V}_{\text {OUT }}$ of the sensor coil is described by the cubic dependency on distance $\mathrm{R}$ between the sensor and actuator, $V_{\text {OUT }} \propto R^{-3}$ [10].

The signal converter proposed in this work is part of complex research dedicated to the development of the Magnetic Tracking System Integrated Development Environment (MTS-IDE). The research has been carried out by our team in a series of commercial projects, which somewhat restricts disclosure of details. MTS-IDE is intended for enhancing the efficacy of parametric optimization and synthesizing firmware of embedded systems implementing magnetic tracking sensors.

The work presents the results of development, modeling, and investigation into a measuring signal converter for magnetic tracking systems. The novelty of the proposed converter consists in a combination of logarithmic amplification methods and synchronous demodulation of the output voltage of sensor coils. Fig. 1 shows the block diagram of the signal converter whose informative signal is the magnetic coupling coefficient between the sensor coil and actuator one. The main blocks of the scheme are Control Unit, Log Amplifier, Synchro Detector, LF (low frequency) Filter, Actuator Driver, ADC (analog-to-digital converter).

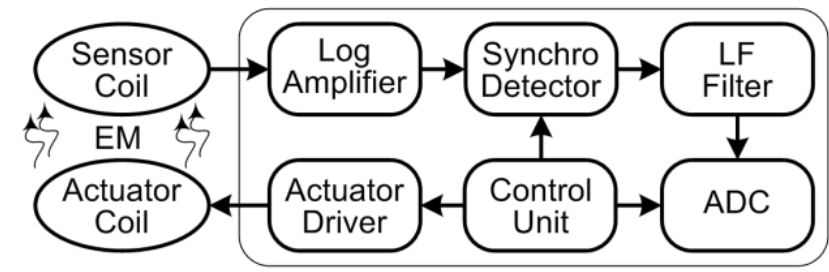

Fig. 1. The block diagram of the measuring signal converter for magnetic tracking systems

Briefly, the signal converter operation can be described as follows. The Control Unit forms a driving signal, which is AC voltage in frequency band $\mathrm{f}_{\mathrm{MT}}$ ranging from $10 \mathrm{~Hz}$ to $500 \mathrm{kHz}$. As is corroborated by literature analysis [18] and our own study, there exist criteria for the selection of the optimal frequency $f_{\text {MT0 }}$ of the driving signal. So, at frequencies $\mathrm{f}_{\mathrm{MT}}<1 \mathrm{kHz}$ the magnetic coupling coefficient in couples "actuator - sensor" is rather small, which makes it necessary to increase the diameter of coils and their number of turns. On the contrary, at frequencies $\mathrm{f}_{\mathrm{MT}}>1 \mathrm{kHz}$ the 
reference magnetic field is getting increasingly distorted by metal items in vicinity, which leads to accuracy deterioration. The Actuator Driver forms the Actuator Coil current using the driving signal.

The Log Amplifier amplifies the AC voltage formed on the Sensor Coil and thus provides compression of its dynamic range. Compression is done by taking the logarithm of the voltage, which exploits the corresponding properties of the volt-ampere characteristics of $\mathrm{p}-\mathrm{n}$ junctions. The schematic of the logarithmic amplifier shown in Fig. 2 incorporates operational amplifier $\mathrm{OA}_{1}$ whose negative-feedback circuit is formed by diodes $\mathrm{D}_{1}$ and $\mathrm{D}_{2}$. The volt-ampere characteristics of $\mathrm{p}-\mathrm{n}$ junctions of these diodes are described by exponential dependencies. Therefore, voltage $\mathrm{V}_{\text {OUT }}$ at the output of the amplifier depends on the logarithm of the input current, $I_{I N}=V_{I N} / R_{1}$, where $V_{\text {IN }}$ is the voltage on sensor coil SC; $R_{1}$ is the resistance of the resistor in the amplifier input circuit. Resistor $R_{2}$ is used for stabilizing the amplifier below some threshold current value, which is defined by parasitic currents in p-n junctions and on a printed board surface. The resistance of $\mathrm{R}_{2}$ is typically chosen within the range $10^{5} \ldots 10^{7} \mathrm{Ohm}$.

Let us analyze the parameters of diodes before designing and verifying the mathematical model of the logarithmic amplifier. We chose diodes of the BAS16 family for the implementation of our signal converter. They are constructed using the SMD (Surface Mount Device) technology and possess a wide current range, high speed, and small-size.

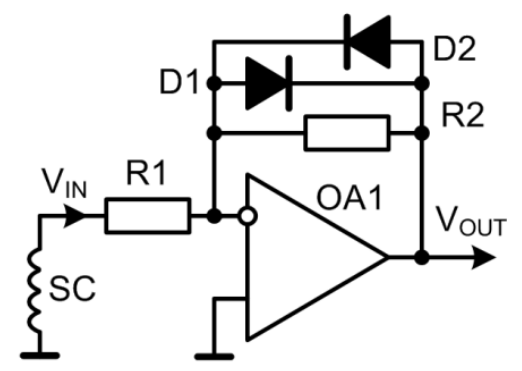

Fig. 2. The schematic of the logarithmic amplifier

Experimental data and the approximated volt-ampere characteristic of the diode BAS16 are shown in Fig. 3. One can observe three distinctive regions. The main region relates to currents within the range from $10^{-6} \mathrm{~A}$ to $10^{-2} \mathrm{~A}$ and is approximated by a logarithmic dependency of voltage on current - Ln approx. Such an approximation is broken if the current is less than the lower threshold caused by parasitic currents or exceeds the upper threshold caused by parasitic resistances of the diode structure.

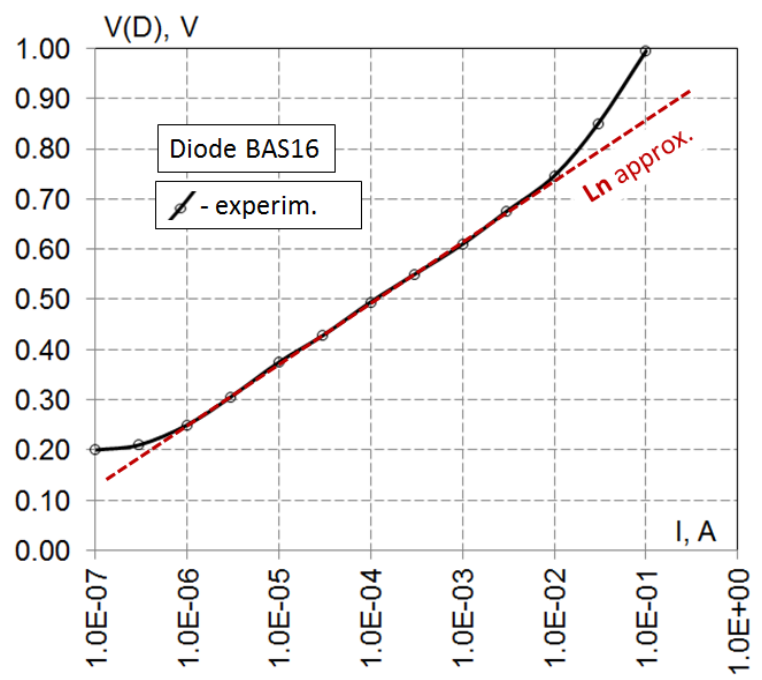

Fig. 3. Experimental data and approximation of the volt-ampere characteristic of the BAS16 diode

Another substantial block of the signal converter is Synchro Detector. It detects the output voltage of the logarithmic amplifier and thus provides an efficient selection of the useful signal out of the noise. This property is achieved by switching the signal polarity in accordance with phase synchronizing pulses which are time-controlled by the driving signal of the Control Unit. The schematic of the synchronous demodulator depicted in Fig. 4 incorporates operational amplifier $\mathrm{OA}_{1}$, whose input circuits are switched by analog two-position switch $\mathrm{SW}_{1}$ and whose negative-feedback circuits are formed by resistors $\mathrm{R}_{1}$ and $\mathrm{R}_{2}$. 


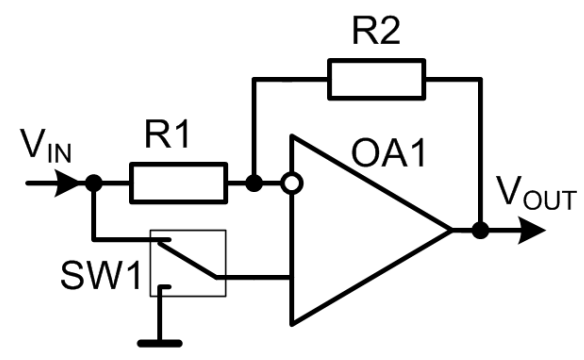

Fig. 4. The schematic of the synchronous demodulator

When the switch is in its upper position (in accordance with the schematic), the non-inverting input of $\mathrm{OA}_{1}$ is connected with the circuit of input voltage $\mathrm{V}_{\mathrm{IN}}$. Assuming that there is no parasitic voltage in the open switch circuit and there is no offset voltage in the operational amplifier, one can claim that no current flows through resistor $\mathrm{R}_{1}$ and, consequently, no voltage drops on the resistor. Therefore, output voltage $\mathrm{V}_{\text {OuT }}$ of the schematic is equal to its input voltage, $\mathrm{V}_{\text {OUT }}=\mathrm{V}_{\mathrm{IN}}$, which implies the input voltage repeater mode. When the switch is in its lower position (in accordance with the schematic), the non-inverting input of $\mathrm{OA}_{1}$ is connected with the common signal point. The negative-feedback circuit holds zero voltage (the common point potential) at the inverting input. Hence, the current flowing through resistor $R_{1}$ is $I_{I N}=V_{I N} / R_{1}$. In this mode the output voltage of the circuit is $V_{\text {OUT }}=-R_{2} \cdot V_{\text {IN }} / R_{1}$, which means the voltage inversion mode, $V_{\text {OUT }}=-V_{\text {IN }}$, provided that $R_{2}=R_{1}$.

Other blocks - LF Filter, Actuator Driver, and ADC - are typical components of most measuring converters. Their parameters and operation are not crucial from the standpoint of the novelty of this work and therefore are not considered here.

\section{Signal Converter Verification}

Simulation of the measuring converter was carried out on the basis of universal and specialized SPICE models of operational amplifiers, diodes, multiplexers and other components in MicroCap. At the first stage, we studied voltampere characteristics of diodes for discrete temperature values ranging from $0^{\circ} \mathrm{C}$ to $100^{\circ} \mathrm{C}$ (Fig. 5). Such a study assumes that the SPICE model of diodes included in the logarithmic amplifier should be verified and specified in accordance with the selected diode type. The main result of this investigation stage is that the actual diode parameters and the temperature coefficients of the diode SPICE model have been brought to an agreement.

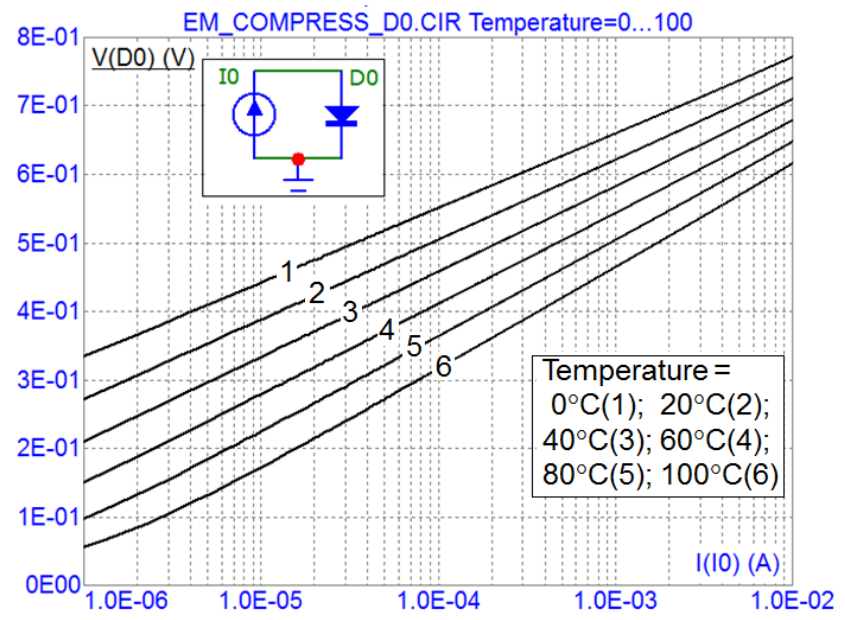

Fig. 5. The dependencies of voltage $V(D 0)$ at the diode on current $I(I 0)$ for the following temperature values: Temperature $=0^{\circ} \mathrm{C}(1) ; 20^{\circ} \mathrm{C}(2) ; 40^{\circ} \mathrm{C}(4) ; 60^{\circ} \mathrm{C}(4) ; 80^{\circ} \mathrm{C}(5) ; 100^{\circ} \mathrm{C}(6)$

At the second stage, we carried out an investigation aimed at the adjustment of the diode SPICE model parameters taking into account the discrepancies between the diode characteristic and the theoretical mathematical dependencies for $\mathrm{p}$-n junctions. For the most part, such discrepancies are attributed to the fact that the diode volt-ampere characteristic is influenced by the emission of charge carriers in the $p-n$ barrier and parasitic resistance of its semiconductor structure. The SPICE model defines emission of charge carriers by coefficient $\mathrm{N}$ (Emission Coefficient), whereas parasitic resistance is set by resistor $\mathrm{R}_{\mathrm{S}}$ (Series Resistance). Fig. 6 shows the simulation results that take into consideration the above-mentioned reasons for discrepancies between the theory and practice for injection coefficients $\mathrm{N}=1.0$ (1), 1.5 (2), 2.0 (3). Fig. 7 depicts the simulation results for discrete values of parasitic diode resistance $\mathrm{R}_{\mathrm{S}}=0$ (1), $10 \mathrm{Ohm}$ (2), $20 \mathrm{Ohm}$ (3), $30 \mathrm{Ohm}$ (4), $40 \mathrm{Ohm}$ (5). The obtained modeling results 
demonstrate discrepancies between the actual volt-ampere characteristic and its nominal logarithmic dependency. The results are in good agreement with the experiments shown in Fig. 3.

At the last stage, we performed integrated simulation dedicated primarily to transient analysis. With this purpose, we synthesized an equivalent circuit of the SPICE model that incorporated both synchro detector and logarithmic amplifier blocks. The transient analysis results for the measuring converter are shown in Fig. 8. The time diagrams of the input signal and its synchronous demodulation were obtained for an input sine wave of frequency $1 \mathrm{kHz}$ and discrete amplitude values E-6 V (1); 1E-5 V (2); 1E-4 V (3); 1E-3 V (4); $1 \mathrm{E}-2 \mathrm{~V}(5) ; 1 \mathrm{E}-1 \mathrm{~V}(6) ; 1 \mathrm{~V}$ (7), with and without taking the logarithm. The obtained results back up the requirements set to synchronous signal demodulation and its highly efficient logarithmic compression.

SPICE simulation results of the proposed measuring converter and the parameters were experimentally verified using a prototype. The converter functionality was studied using oscillographic methods (Fig. 9). Its parameters were measured using the above-mentioned MTS-IDE - Magnetic Tracking System Integrated Development Environment (Fig. 10).

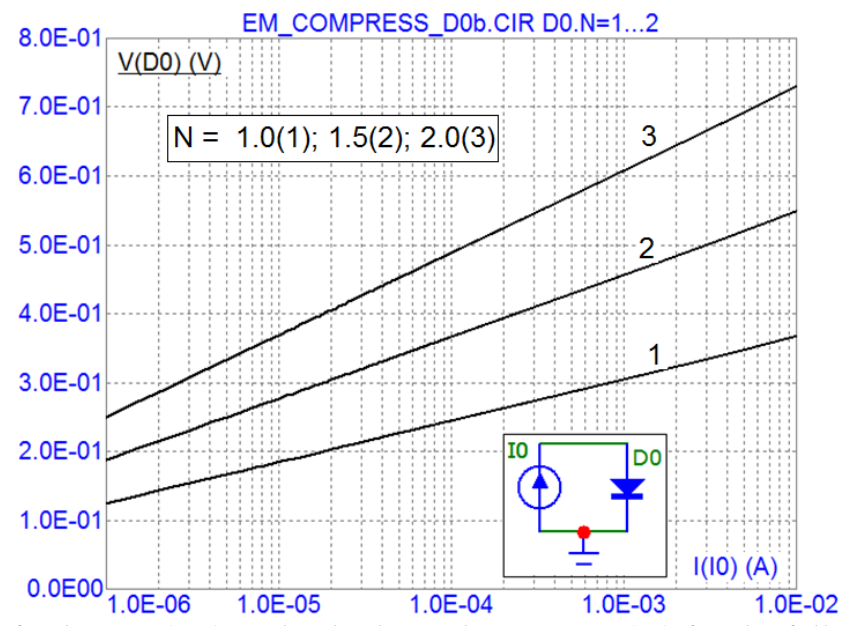

Fig. 6. The dependencies of voltage V(D0) at the diode on the current I(I0) for the following injection coefficients: $N=1.0(1) ; 1.5(2) ; 2.0(3)$

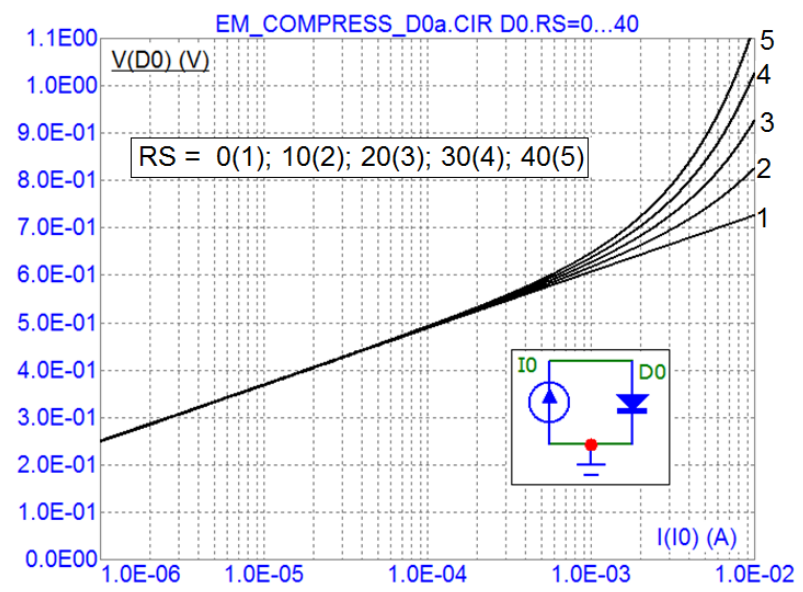

Fig. 7. The dependencies of voltage $V(D 0)$ at the diode on the current $I(I 0)$ for the following parasitic diode resistance values: $R S=0$ (1); $10 \mathrm{Ohm} \mathrm{(2);} 20 \mathrm{Ohm} \mathrm{(3);30} \mathrm{Ohm} \mathrm{(4);} 40 \mathrm{Ohm} \mathrm{(5)}$ 

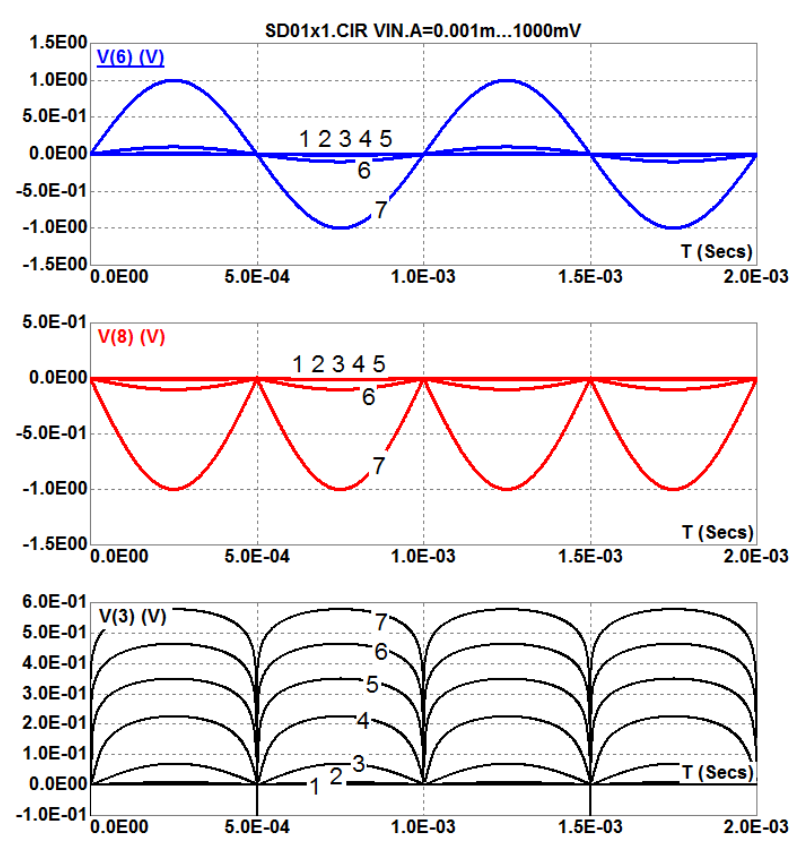

Fig. 8. The time diagrams of the input signal (on the top) and the results of synchronous demodulation without (in the middle) and with (at the bottom) taking the logarithm

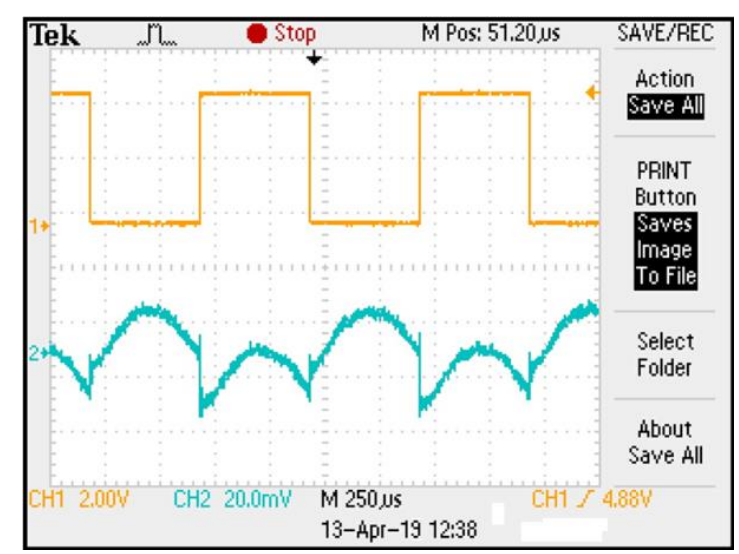

Fig. 9. Sample oscillograms of sync pulses (on the top) and signals at the output of the synchronous demodulator (at the bottom)

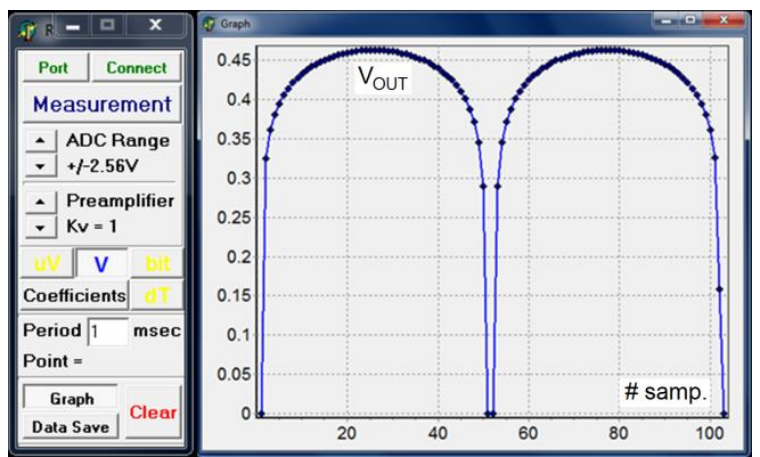

Fig. 10. The result of an experimental investigation into signals at the converter output using MTS-IDE

The analog-to-digital converter of MTS-IDE is implemented upon the micro converter ADuC834 with a word length of 24 bits. Our experiments have proven that the dynamic range of noise-immune signal conversion covers six orders of magnitude, from $1 \mathrm{E}-6 \mathrm{~V}$ to $1 \mathrm{~V}$. The obtained results are of key importance for further enhancement of magnetic tracking systems in general and expansion of their measurement volumes while preserving noise-immunity in particular.

\section{Conclusion}

The work presents the results of developing a logarithmic measuring converter for magnetic tracking systems. It has been shown that one of the problems crucial for further enhancement of magnetic tracking systems is a noise-immune measurement of signals from sensor coils in a wide measurement range. More precisely, the measurement range from 
several microvolts to hundreds of millivolts is required. The fisrt values correspond to distances of several meters in couples "actuator - sensor". The latter ones relate to the case when the distances reduce to several centimeters.

The novelty of the proposed measuring signal converter for magnetic tracking systems consists in a combination of logarithmic amplification methods and synchronous demodulation of the output voltage of sensor coils. Voltage logarithm compression has been performed upon volt-ampere characteristics of semiconductor $p$ - $n$ junctions. The synchronous demodulator provides an efficient selection of the useful signal from electromagnetic noise. Simulation and experiments have proved that the dynamic range of noise-immune signal measurement using the developed converter covers six orders of magnitude, from $1 \mathrm{E}-6 \mathrm{~V}$ to $1 \mathrm{~V}$. The characteristics of the proposed solution have been measured with the above-mentioned MTS-IDE. Its functionality was investigated with oscillograph methods. The obtained results are of key importance for further improvement of magnetic tracking systems, particularly for their noise-immune measurement volume expansion.

\section{Conflict of Interest}

The authors claim that there are no possible financial or other conflicts over the work.

\section{References}

[1] W. Hongtao, Y. Zhimin, W. Ping, B. Santoso, O. Lian, "A novel method of motion tracking for virtual reality using magnetic sensors", in Asia-Pacific Magnetic Recording Conference (APMRC-2018), Shanghai, 2018.

DOI: 10.1109/APMRC.2018.8601108.

[2] M. Singh and B. Jung, "High-definition wireless personal area tracking using AC magnetic field for virtual reality", in 2017 IEEE Virtual Reality (VR), Los Angeles, 2017.

DOI: 10.1109/VR.2017.7892250.

[3] D. Fedasyuk, R. Holyaka, and T. Marusenkova, "A tester of the MEMS accelerometers operation modes", in 2019 3rd Int. Conf. on Advanced Information and Communications Technologies (AICT), Lviv, 2019.

DOI: 10.1109/aiact.2019.8847840.

[4] D. Fedasyuk, R. Holyaka, and T. Marusenkova, "Method of analyzing dynamic characteristics of MEMS gyroscopes in test measurement mode", in 2019 9th Int. Conf. on Advanced Computer Information Technologies (ACIT), Ceske Budejovice, 2019, pp. 157-160.

DOI: $10.1109 /$ acitt.2019.8780058.

[5] D. Jo and G. Kim, "ARIoT: scalable augmented reality framework for interacting with Internet of Things appliances everywhere", IEEE Trans. Consum. Electron., vol. 62, no. 3, pp. 334-340, Aug. 2016.

DOI: 10.1109/tce.2016.7613201.

[6] T. Reichl, J. Gardiazabal, and N. Navab, "Electromagnetic Servoing - a new tracking paradigm", IEEE Trans. Med. Imag., vol. 32, no. 8, pp. 1526-1535, Aug. 2013.

DOI: $10.1109 /$ tmi.2013.2259636.

[7] A. Franz et al., "Electromagnetic tracking in medicine - a review of technology, validation, and applications", IEEE Trans. Med. Imag., vol. 33, no. 8, pp. 1702-1725, May 2014.

DOI: $10.1109 /$ tmi.2014.2321777.

[8] N. Alves et al., "An MEG-compatible electromagnetic-tracking system for monitoring orofacial kinematics", IEEE Trans. Biomed. Eng., vol. 63, no. 8, pp. 1709-1717, Nov. 2015.

DOI: 10.1109/tbme.2015.2500102.

[9] S. Song, Z. Li, H. Yu, and H. Ren, "Electromagnetic positioning for tip tracking and shape sensing of flexible robots", IEEE Sensors J., vol. 15, no. 8, pp. 4565-4575, Aug. 2015.

DOI: $10.1109 /$ jsen.2015.2424228.

[10] Pérez et al., VR EM Motion tracking systems \& applications. Málaga, Spain: PREMO S.L., 2017.

[11] A. Matthews, "VR 3D electromagnetic motion tracking sensor", 2017. [Online]. Available: https://www.electronicspecifier.com/sensors/vr-3d-electromagnetic-motion-tracking-sensor\#downloads

[12] I. Skog, "Inertial and magnetic-field sensor arrays - capabilities and challenges", in 2018 IEEE SENSORS, New Delhi, India, Oct. 2018.

DOI: $10.1109 /$ icsens.2018.8589760.

[13] H. Dai, S. Song, C. Hu, B. Sun, and Z. Lin, " Novel 6-D tracking method by fusion of 5-D magnetic tracking and 3-D inertial sensing", IEEE Sensors J., vol. 18, no. 23, pp. 9640-9648, Dec. 2018.

DOI: 10.1109/JSEN.2018.2872650.

[14] W. Kim, J. Song, and F. Park, "Closed-form position and orientation estimation for a three-axis electromagnetic tracking system", IEEE Trans. Ind. Electron., vol. 65, no. 5, pp. 4331-4337, May 2018.

DOI: $10.1109 /$ tie.2017.2760244.

[15] H. He, P. Maheshwari, and D. Pommerenke, "The development of an EM-field probing system for manual nearfield scanning", IEEE Trans. Electromagn. Compat., vol. 58, no. 2, pp. 356-363, Apr. 2016.

DOI: $10.1109 /$ temc.2015.2496376.

[16] H. Sadjadi, K. Hashtrudi-Zaad, and G. Fichtinger, "Simultaneous electromagnetic tracking and calibration for dynamic field distortion compensation", IEEE Trans. Biomed. Eng., vol. 63, no. 8, pp. 1771-1781, Aug. 2016.

DOI: $10.1109 /$ tbme.2015.2502138.

[17] I. Sharp, K. Yu, and T. Sathyan, "Positional accuracy measurement and error modeling for mobile tracking", IEEE 
Trans. Mobile Comput., vol. 11, no. 6, pp. 1021-1032, June 2012.

DOI: $10.1109 /$ tmc.2011.119.

[18] P. Ripka and A. Zikmund, "Magnetic tracker with high precision", Procedia Engineering, vol. 25, pp. 1617-1620, Dec. 2011.

DOI: $10.1016 /$ j.proeng.2011.12.400. 